University of New Hampshire

University of New Hampshire Scholars' Repository

$5-1994$

\title{
Application of a maximum likelihood processor to acoustic backscatter for the estimation of seafloor roughness parameters
}

Zoi-Heleni Michalopoulou

Duke University

Christian de Moustier

University of California - San Diego

Follow this and additional works at: https://scholars.unh.edu/ccom

Part of the Oceanography and Atmospheric Sciences and Meteorology Commons

\section{Recommended Citation}

Z.-H. Michalopoulou, D. Alexandrou, and C. de Moustier, 'Application of a maximum likelihood processor to acoustic backscatter for the estimation of seafloor roughness parameters', The Journal of the Acoustical Society of America, vol. 95, no. 5, p. 2467, 1994.

This Journal Article is brought to you for free and open access by the Center for Coastal and Ocean Mapping at University of New Hampshire Scholars' Repository. It has been accepted for inclusion in Center for Coastal and Ocean Mapping by an authorized administrator of University of New Hampshire Scholars' Repository. For more information, please contact Scholarly.Communication@unh.edu. 


\title{
Application of a maximum likelihood processor to acoustic backscatter for the estimation of seafloor roughness parameters
}

\author{
Zoi-Heleni Michalopoulou and Dimitri Alexandrou \\ Department of Electrical Engineering, Duke University, Durham, North Carolina 27708-0291 \\ Christian de Moustier ${ }^{a)}$ \\ Marine Physical Laboratory, Scripps Institution of Oceanography, University of California, San Diego, \\ La Jolla, California 92093-0205
}

(Received 30 April 1993; accepted for publication 4 January 1994)

\begin{abstract}
Maximum likelihood (ML) estimation is used to extract seafloor roughness parameters from records of acoustic backscatter. The method relies on the Helmholtz-Kirchhoff approximation under the assumption of a power-law roughness spectrum and on the statistical modeling of bottom reverberation. The result is a globally optimum, highly automated technique that is a useful tool in the context of seafloor classification via remote acoustic sensing. The general geometry of the Sea Beam bathymetric system is incorporated into the design of the ML processor in order to make it applicable to real acoustic data collected by this system. The processor is initially tested on simulated backscatter data and is shown to be very effective in estimating the seafloor parameters of interest. The simulated data are also used to study the effect of data averaging and normalization in the absence of system calibration information. The same estimation procedure is applied to real data collected over two central North Pacific seamounts, Horizon Guyot and Magellan Rise. The Horizon Guyot results are very close to estimates obtained through a curve-fitting procedure presented by de Moustier and Alexandrou [J. Acoust. Soc. Am. 90, 522-531 (1991)]. In the case of Magellan Rise, discrepancies are observed between the results of ML estimation and curve fitting.
\end{abstract}

PACS numbers: $43.30 . \mathrm{Gv}, 43.30 . \mathrm{Hw}, 43.30 . \mathrm{Pc}$

\section{INTRODUCTION}

The objective of this paper is to derive a methodology for estimating seafloor roughness spectrum parameters based on the angular dependence function of acoustic backscatter. This is an important step toward the longrange goal of automated seafloor classification using remote acoustic sensing. Heretofore, this problem has received relatively little attention in the literature. A first attempt, by de Moustier and Alexandrou, ${ }^{1}$ was based on fitting intensity curves estimated from real data to theoretical scattering strength curves obtained from the Helmholtz-Kirchhoff formulation as developed by Jackson et al. $^{2}$ This approach relied on a qualitative "goodness-offit" criterion and could not be easily automated. Here, we introduce a quantitative, globally optimum, and highly automated technique for performing the same task. Another quantitative approach to the problem of seafloor parameter estimation is by Matsumoto et $a l^{3}$

The selection of an appropriate criterion is an issue of primary importance in any estimation problem. A simple and popular choice is the minimum mean-squared error (MMSE) criterion, which yields estimates that minimize the distance (i.e., squared error) between measurements and theoretical predictions. However, MMSE estimation can lead to inconsistent results because it does not include any information concerning the probabilistic structure of

a) On leave at the Naval Research Laboratory, Code 7420, Washington, DC 20375-5350. the observed data. ${ }^{4}$ Alternatively, the maximum likelihood (ML) criterion can be employed, which takes explicit account of the statistical character of the received signal. For a given set of data, MMSE leads to the same results regardless of the underlying distributions, whereas the performance of ML-based estimators relies on these distributions. If the statistical modeling is done correctly, the ML approach produces more reliable estimates by taking advantage of existing knowledge concerning the nature of the observed data. For this reason, ML is the criterion of choice here. Our ML estimation technique is based on the following assumptions: First, the received reverberation quadrature components are normally distributed. Equivalently, the magnitude of the complex reverberation envelope is Rayleigh distributed and the instantaneous intensity is governed by a scaled $\chi^{2}$ distribution with two degrees of freedom. Second, the expected value of the received backscattered intensity is assumed to be of the form predicted by the Helmholtz-Kirchhoff model as developed by Jackson et $\mathrm{l}^{2}{ }^{2}$ In this manner, our method combines elements of two well-known scattering models (point-scattering and Helmholtz-Kirchhoff) into a unified statistical framework where the ML criterion can be applied. The result is an efficient tool yielding globally optimum, reliable parameter estimates. In addition, the obtained likelihood surfaces offer useful insight into the uncertainty associated with this estimation problem.

As in de Moustier and Alexandrou, ${ }^{1}$ the estimation process is based on relating measurements of seafloor 
acoustic backscatter to a Helmholtz-Kirchhoff formulation of the reverberation process based on a power-law roughness spectrum. ${ }^{2}$ The selection of the particular scattering model is dictated by the range of angles of incidence we are interested in, which is $0^{\circ}-20^{\circ}$, as will be discussed later. For this angular range, the Kirchhoff approximation is considered preferable to other approaches. ${ }^{2}$

To test the proposed ML method, simulated backscatter data were created, based on the statistical model described above. This allows us to investigate the performance of the method in a controlled environment, giving us insight into the sensitivity of the method to variations in model parameters and hence into its ability to discriminate between different seafloor types. In addition, it allows us to study the effect of a normalization procedure that must often be applied to real data because of the absence of system calibration information. Following testing with simulated data, the ML method is applied to real acoustic data.

The paper is organized as follows: The HelmholtzKirchhoff formulation as developed by Jackson et $a l^{2}$ is briefly discussed in Sec. I. Section II describes the statistical model of backscattered intensity leading to the development of the ML estimator, which is presented in Sec. III. The performance of the ML processor with simulated data is presented in Sec. IV A, and Sec. IV B presents the estimation results obtained with data collected with the Sea Beam system over two central North Pacific seamounts.

\section{THE ACOUSTIC MODEL}

Backscattering strength $\sigma$ is a parameter of bottom reverberation, which, on a logarithmic scale, is defined as ${ }^{5}$

$$
S=10 \log _{10} \sigma=10 \log _{10}\left(I_{\text {scat }} / I_{\text {inc }}\right),
$$

where $I_{\text {scat }}$ is the intensity of sound scattered by a unit area of the seafloor, measured at a unit distance from the area, when the seafloor is insonified by a plane wave of intensity $I_{\text {inc }}$.

In Jackson's model, ${ }^{2}$ the two main components contributing to the total backscatter are interface roughness and sediment volume scattering. The Kirchhoff approximation is employed for the calculation of backscattering strength due to the roughness of the water-sediment interface, and it is assumed that the spatial power spectrum can be modeled in a power-law form. ${ }^{6}$ In terms of the spatial wave number $k$, the spectrum can be written as

$$
W(k)=\beta k^{-\gamma},
$$

where $\gamma$ takes values in the interval 3-3.5 for highfrequency bottom backscatter. ${ }^{1,2}$ Parameter $\beta$ is the slope of the power spectrum; it has been found to take values on the order of $10^{-5}$ (in mks units) for sedimented regions over Horizon Guyot and Magellan Rise in the central North Pacific ${ }^{1}$ and on the order of $10^{-4}$ for tectonic or volcanic terrains. $^{3}$
The morphology of the seafloor is described by the structure function $D(r)$, which expresses the mean square height difference of the seafloor for a specific horizontal distance $r$ and can be expressed as

$$
D(r)=C_{h}^{2} r^{2 \alpha} \text {. }
$$

The quantity $C_{h}$ is defined as

$$
C_{h}^{2}=\left[2 \pi \beta \Gamma(2-\alpha) 2^{-2 \alpha}\right] /[\alpha(1-\alpha) \Gamma(1+\alpha)],
$$

where $\Gamma$ is the gamma function and $\alpha=\gamma / 2-1$.

The backscattering strength $\sigma$ can be written as ${ }^{2}$

$$
\begin{aligned}
\sigma(\alpha, \beta, \theta)= & \frac{g^{2}(0)}{8 \pi \sin ^{2}(\pi / 2-\theta) \cos ^{2}(\pi / 2-\theta)} \\
& \times \int_{0}^{\infty} \exp \left(-q u^{2 \alpha}\right) J_{0}(u) u d u,
\end{aligned}
$$

where

$$
q=\sin ^{2}(\pi / 2-\theta) \cos ^{-2 \alpha}(\pi / 2-\theta) C_{h}^{2} 2^{1-2 \alpha} k_{a}^{2(1-\alpha)},
$$

$g(0)$ is the plane wave reflection coefficient at vertical incidence $(\theta=0), J_{0}$ is the zeroth-order Bessel function of the first kind, $k_{a}$ is the acoustic wave number, and $\theta$ is the angle of incidence.

At vertical incidence, Eq. (5) is indeterminate. For $\theta=0$, the backscattering strength is defined as ${ }^{1}$

$$
\sigma(\alpha, \beta, 0)=g^{2}(0) C_{h}^{-2 / \alpha}\left(2 k_{a}^{2}\right)^{(\alpha-1) / \alpha} \Gamma(1 / \alpha)(8 \pi \alpha)^{-1} .
$$

The potential contribution of volume scattering is not taken into account, because it is desired to keep the computational requirements of the proposed estimation process manageable.

\section{THE STATISTICAL MODEL}

The backscattered signal must ultimately be viewed as a random process. The "physical" model (i.e., the Helmholtz-Kirchhoff approximation) described in Sec. I provides a single second-order statistic of the random process, backscattering strength, in terms of two physically meaningful seafloor parameters ( $\alpha$ and $\beta$ ). However, it offers no clues as to the probability density function (pdf) governing the random process. An alternative view of reverberation is given by the point-scattering model. ${ }^{7}$ According to this model, the scattered signal results from the linear superposition of the individual echoes emanating from a large number of point reflectors distributed independently on a plane surface. Although this model has a relatively weak physical connection, it has been shown to accurately predict probability distributions. ${ }^{7-9}$ Here, we create a unified statistical framework consistent with both the Helmholtz-Kirchhoff and the point-scattering model. This framework will be used both to simulate backscatter and to formulate the ML estimator.

It is assumed here that reverberation consists of a random (incoherent) component only. A coherent component usually appears in the near-specular returns; it is consid- 
ered negligible compared to the incoherent field, when the rough surface relief is greater or comparable to the acoustic wavelength. ${ }^{2}$ For typical values of $\alpha$ and $\beta$ (0.60 and $4.0 \times 10^{-5}$, respectively), the $\mathrm{mm}$ height difference calculated from Eq. (5) for a horizontal range of $20 \mathrm{~m}$ is equal to $12.8 \mathrm{~cm}$. For a frequency of $12 \mathrm{kHz}$, which is the operating frequency at which the available data have been collected, the wavelength is approximately $12 \mathrm{~cm}$. The roughsurface relief is then comparable to the acoustic wavelength, thus allowing the assumption that the coherent component is negligible. ${ }^{2}$ Additional support for this assumption was provided by estimates of coherent intensity obtained from real data by calculating the squared mean of the scattered field, which indicated that this coherent term is three orders of magnitude smaller than the total average intensity.

In the absence of a coherent component, the pointscattering model predicts that the envelope of bottom reverberation is in the limit a complex Gaussian random variable (rv). The real and imaginary parts are independent and they have zero mean and equal variances. ${ }^{7-9}$ Equivalently, the reverberation magnitude (often referred to as "the envelope") is modeled as a Rayleigh rv and reverberation magnitude squared is modeled as a $\chi^{2}$ rv with 2 degrees of freedom (dof). Average intensity is defined as the expected value of the reverberation magnitude squared. Because our objective is to interpret the angular dependence of the backscattered intensity according to the acoustic model described in Sec. I, we focus our attention on the statistical behavior of reverberation magnitude squared.

$$
\text { A } \chi^{2} \text { pdf with } v \text { dof is given by }
$$

$f_{y}(Y)=\left[1 / 2^{v / 2} \Gamma(v / 2)\right] Y^{(v / 2)-1} \exp (-Y / 2), \quad Y>0$,

with $E[Y]=v$ and $\operatorname{VAR}[Y]=2 v$.

Let $Z_{I}$ and $Z_{Q}$ be two independent, normally distributed rv's representing the real and imaginary parts of the received reverberation process. The fact that the coherent component is assumed to be negligible implies a zero mean for both rv's. In addition, $Z_{I}$ and $Z_{Q}$ have equal variances. Let us for the moment assume that their variance is equal to 1 . Then, the pdf of the reverberation magnitude squared $\left(Z_{I}^{2}+Z_{Q}^{2}\right)$ is of the form of Eq. (8) with $v=2$. Of course, the variance $\phi^{2}$ will not, in general, be equal to 1 . We can account for this by creating a scaled rv defined as

$$
X=\phi^{2}\left(Z_{I}^{2}+Z_{Q}^{2}\right)=\phi^{2} Y,
$$

where $Y$ is a $\chi^{2}$ rv of the form of Eq. (8) with 2 dof. This rv $X$ is now used to model the squared magnitude of the received reverberation signal.

Using Eqs. (8) and (9), the pdf of $X$ can be written as

$$
f_{x}(X)=\frac{1}{\phi^{2}} f_{y}\left(\frac{X}{\phi^{2}}\right)=\frac{1}{2 \phi^{2}} \exp \left(-\frac{X}{2 \phi^{2}}\right), \quad X>0,
$$

with $E[X]=2 \phi^{2}$ and $\operatorname{VAR}[X]=4 \phi^{4}$.

According to Eq. (1), backscattering strength is an expression of normalized average backscattered intensity. Then, for a particular angle of incidence $\theta_{i}$, backscattering strength as given by Eqs. (5) and (7) is the expected value of a rv $X_{i}$, distributed according to Eq. (10). It follows that

$$
E\left[X_{i}\right]=2 \phi_{i}^{2}=\sigma_{i},
$$

where $X_{i}=X\left(\theta_{i}\right), \phi_{i}=\phi\left(\alpha, \beta, \theta_{i}\right)$, and $\sigma_{i}=\sigma\left(\alpha, \beta, \theta_{i}\right)$. Thus the pdf of the received reverberation magnitude squared [Eq. (10)] is fully described by the backscattering strength for a given set of parameters $\alpha, \beta$, and $\theta_{i}$.

\section{THE ML ESTIMATOR}

The mathematical framework developed in Secs. I and II is now used to develop an optimum ML processor. It is assumed that the data consist of $n$ acoustic backscattered returns obtained at $m$ different angles of incidence. Only the squared magnitude of each return is preserved. For an angle of incidence $\theta_{i}, i=1, \ldots, m$, this quantity is described by a rv $X_{i}$ distributed according to Eq. (10). Assuming that the returns from different angles of incidence are independent, the joint distribution of the random vector $\bar{X}=\left[X_{1}, X_{2}, \ldots, X_{m}\right]^{T}$ (where $[\cdot]^{T}$ stands for the transpose of $[\cdot])$ is a product of the marginal one-dimensional $\chi^{2}$ distributions (i.e., the individual distributions corresponding to each angle $\theta_{i}$ ). For $n m$-dimensional observations of $\bar{X}$, the likelihood function $L\left(\bar{\phi}^{2}\right)$, where $\bar{\phi}^{2}=\left[\phi_{1}^{2}, \phi_{2}^{2}, \ldots\right.$, $\left.\phi_{m}^{2}\right]^{T}$, is given by

$$
L\left(\bar{\phi}^{2}\right)=\prod_{i=1}^{m} l\left(\phi_{i}^{2}\right),
$$

where

$$
l\left(\phi_{i}^{2}\right)=\frac{1}{2^{n} \phi_{i}^{2 n}} \exp \left(-\frac{\Sigma_{j=1}^{n} x_{i j}}{2 \phi_{i}^{2}}\right) .
$$

Parameter $\phi_{i}^{2}$ is related to $X_{i}$ through Eq. (11), and $x_{i j}$ is the $j$ th acoustic observation at the $i$ th angle, $j=1, \ldots, n$, $i=1, \ldots, m$.

The ML estimates for $\alpha$ and $\beta$ could then be calculated by finding the values of $\alpha$ and $\beta$ for which the likelihood function, or equivalently its logarithm, reaches a maximum. This procedure requires a tranformation relating $\alpha$ and $\beta$ to backscattering strength, or equivalently, to parameters $\phi_{i}^{2}$. This transformation is readily available through Eqs. (5) and (7). Specifically, we form a rectangular grid having $\alpha$ and $\beta$ as its coordinates; every point on the grid corresponds to a scattering strength vector $\bar{\sigma}$ calculated at the angle vector $\bar{\theta}=\left[\theta_{1}, \ldots, \theta_{m}\right]^{T}$. Here, $\theta_{i}=\frac{4}{3}+\frac{8}{3}(i-1)$ degrees for $i=1, \ldots, 8$. These specific values were selected because they correspond to the nominal beam steering angles of the Sea Beam bathymetric system. The sonar platform is assumed to be fixed and refraction effects are neglected so that beam steering angles are angles of incidence on a flat bottom. Knowledge of $\bar{\sigma}$ leads to $\bar{\phi}^{2}$ through Eq. (11). This, combined with the acoustic observations $x_{i j}$, allows the calculation of the likelihood function through Eq. (12) for each grid point. Finding the maximum of the resulting ML surface yields the ML estimates of $\alpha$ and $\beta$ for the given set of acoustic observations. The computational load of this method depends only on 


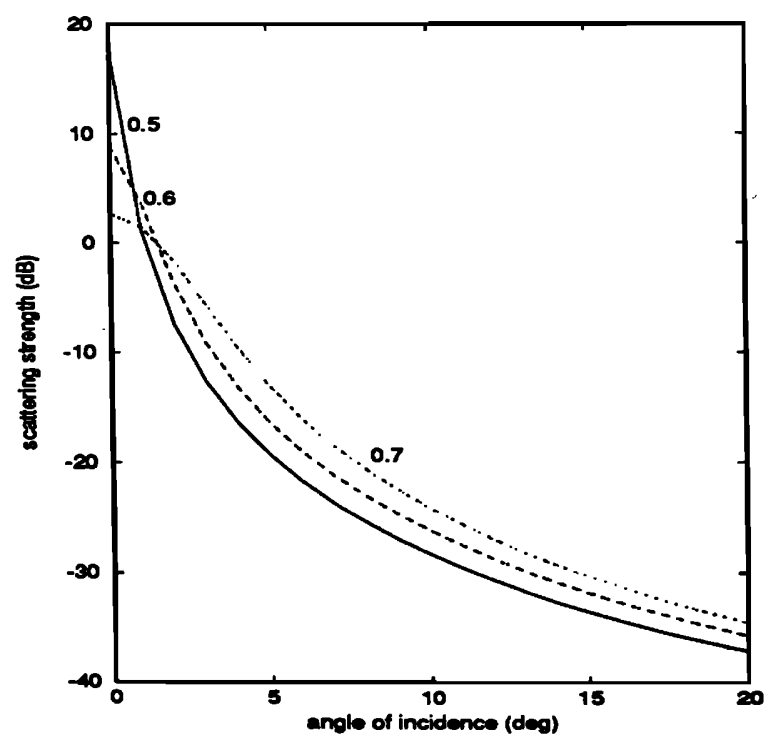

(a)

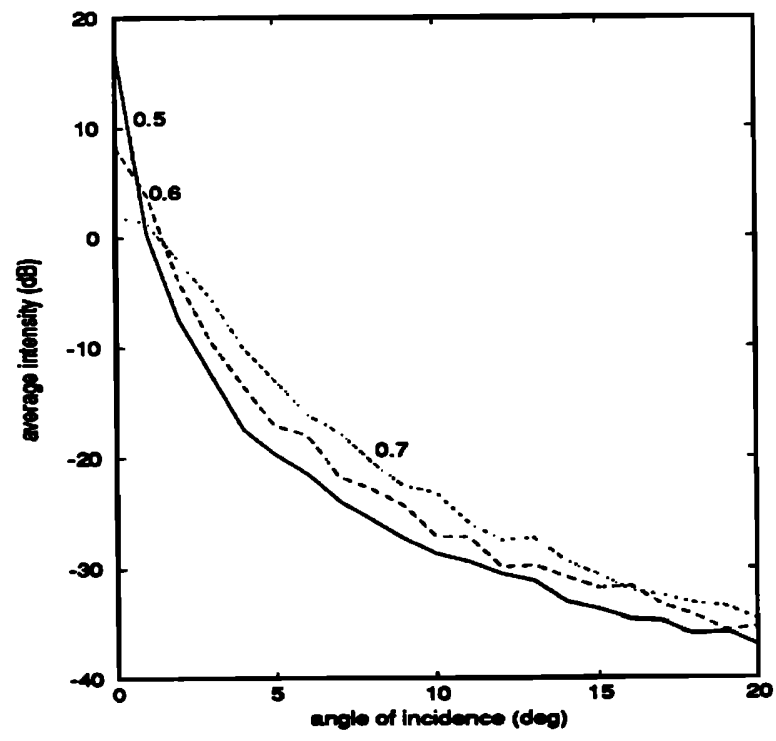

(b)

FIG. 1. (a) Backscattering strength as obtained by Eqs. (5) and (7) for $\alpha=0.5,0.6$, and 0.7 and $\beta$ such that $C_{h}=10^{2 \alpha-3} m^{(1-\alpha)}$; (b) average simulated backscattered intensity for the same parameters.

the cell size of the grid. In addition, it is important to define the ranges in which $\alpha$ and $\beta$ are allowed to vary. Parameter $\alpha$ is chosen to be in the range $[0.4883,0.73]$ and $\beta$ is constrained to be of the order of $10^{-5}$ for the seafloor regions of interest. The ranges for $\alpha$ and $\beta$ are selected on the basis of the existing $a$ priori information. ${ }^{1-3}$

\section{SEAFLOOR PARAMETER ESTIMATION}

\section{A. Simulated data}

Before we apply the ML estimator to real data, we test it with simulated data produced according to the statistical model described in Sec. II. Observations of instantaneous intensity are simulated by drawing samples from the distribution of Eq. (10) for selected values of roughness spectrum parameters and angles of incidence. Figure 1(a) shows the (logarithmic) curves obtained by Eqs. (5) and (7) for $\alpha$ equal to $0.5,0.6$, and 0.7 and angles of incidence in the range $\left[0,20^{\circ}\right]$. The quantity $C_{h}$ was set to $10^{2 \alpha-3}$ in units of meters ${ }^{(1-\alpha)}$. Figure $1(b)$ presents the curves obtained for the same parameters by averaging over 100 sample vectors from scaled $\chi^{2}$ distributions with 2 dof, as described above. It can be seen that the simulation procedure closely approximates the theoretical backscattering strength curves. Since the simulated data obey exactly all model assumptions, we expect the results presented in this section to represent an upper bound of performance for the ML estimator.

A factor that is likely to be of considerable importance in a real-world experiment is the number of observations (pings) available for each estimate. Because the seafloor is highly inhomogeneous, it would be desirable to utilize the smallest possible number of observations. The simulations afford us the opportunity to quantify the significance of this factor in the absence of modeling uncertainties. In addition, they allow us to study the impact of a data normalization scheme, which must often be used in the absence of calibration information for the particular sonar system used during the experiment.

Table I presents the results obtained by using a ( 30 $\times 20)$ grid, with $0.4883 \leqslant \alpha \leqslant 0.73$ and $5.0 \times 10^{-6} \leqslant \beta$ $\leqslant 1.0 \times 10^{-4}$. Simulations were performed with 10,50 , and 100 observations for different combinations of parameters $\alpha$ and $\beta$. Taking into account that ML estimation is performed on a digitized set of parameters, the real estimates in a continuous domain could be located in areas including the grid points given in Table I and their eight nearest neighbors. Figure 2 shows the likelihood surfaces, normalized with respect to their maxima, versus $\alpha$ and $\beta$ for different numbers of observations, for data generated with $\alpha=0.53$ and $\beta=2.0 \times 10^{-5}$. As expected, a larger observation set results in a more peaked likelihood and a decrease in the uncertainty surrounding the main mode. The existence of significant secondary modes indicates a potential difficulty in determining the most likely values for $\alpha$ and $\beta$. Because of the observed multimodality, the implementation of "hill-climbing" techniques for finding the maximum of the likelihood surface, instead of the grid-based method used here, could prove troublesome, since the optimization processes could easily get trapped in local extrema. Finally, the existence of a number of modes indicates that the presentation of only point estimates is not particularly meaningful. A measure of uncertainty, providing a synoptic view of all available information pertaining to the parameters of interest, should accompany the point estimates. Here, this role is performed by the likelihood surface. It 
TABLE I. ML estimates for $\alpha$ and $\beta$ obtained from simulated backscatter with a $\chi^{2}$ modeling with 2 dof for 10,50 , and 100 observations.

\begin{tabular}{cccc}
\hline \hline & \multicolumn{3}{c}{ Maximum likelihood estimation of $\alpha$ and $\beta$} \\
\cline { 2 - 4 } Sample size & $\alpha=0.530$ & $\alpha=0.680$ & $\alpha=0.705$ \\
& $\beta=2.0 \times 10^{-5}$ & $\beta=4.0 \times 10^{-5}$ & $\beta=9.0 \times 10^{-5}$ \\
\hline 10 & 0.5383 & 0.7050 & 0.6217 \\
& $2.0 \times 10^{-5}$ & $5.0 \times 10^{-5}$ & $7.5 \times 10^{-5}$ \\
50 & 0.5217 & 0.6967 & 0.7300 \\
& $2.0 \times 10^{-5}$ & $4.5 \times 10^{-5}$ & $1.0 \times 10^{-4}$ \\
100 & 0.5300 & 0.6883 & 0.7133 \\
& $2.0 \times 10^{-5}$ & $4.0 \times 10^{-5}$ & $9.5 \times 10^{-5}$ \\
\hline \hline
\end{tabular}

should also be noted, that the relative location of the multiple modes on the grid reveals the high degree of correlation between $\alpha$ and $\beta$. The exact nature of the correlation pattern is governed by the highly nonlinear function relating $\phi_{i}^{2}$ to $\alpha$ and $\beta$ [i.e., Eq. (5)].

The results presented thus far are based on modeling the received mean backscattered intensity as scattering strength calculated by Eq. (5). However, in a real sonar deployment, it is often the case that reliable system calibration information is not available. This prohibits the calculation of backscattering strength from measurements of backscattered intensity. Therefore, it is necessary to develop a normalization scheme to establish a correspondence between real measurements and theoretical predictions.

The normalization procedure used here is based on the introduction of a new $\mathrm{rv} W_{i}$. Let $W_{i}=X_{i} / \lambda^{2}$, where $X_{i}$ is a rv distributed according to $\mathrm{Eq}$. (10) and corresponds to the backscattered intensity for the angle of incidence $\theta_{i}$, where $i=1, \ldots, 8$, and $\lambda$ is a normalization factor. Then, the pdf $f_{w_{i}}$ of $W_{i}$ is

$$
\begin{aligned}
f_{w_{i}}\left(W_{i}\right) & =\frac{\lambda^{2}}{2 \phi_{i}^{2}} \exp \left(-\frac{W_{i} \lambda^{2}}{2 \phi_{i}^{2}}\right) \\
& =\frac{1}{2\left(\phi_{i} / \lambda\right)^{2}} \exp \left(-\frac{W_{i}}{2\left(\phi_{i} / \lambda\right)^{2}}\right), \quad W_{i}>0 .
\end{aligned}
$$

The normalization factor is chosen to be the average intensity received at the angle of incidence closest to nadir (here $\theta_{1}=\frac{40}{3}$ ). Thus the expected value of $W_{1}$ should be

$$
E\left[W_{1}\right]=2\left(\phi_{1} / \lambda\right)^{2}=1 \text {. }
$$

Recalling Eq. (11) and the definition of $W_{i}$, this means that $\lambda^{2}=\sigma_{1}=\sigma\left(\alpha, \beta, \frac{40}{3}\right)$. Thus the synthetic data consist of observations of the rv's $X_{i}$ scaled to $W_{i}$ by division with $\sigma_{1}$.

The results of the normalization procedure are presented in Table II and Fig. 3. The likelihood surfaces (Fig. 3) now spread over a broader part of the grid compared to those of Fig. 2. This increase in ambiguity was expected since, following normalization, estimation is based only on the general form of the dependence of backscattered intensity on the angle of incidence and parameters $\alpha$ and $\beta$ while it ignores the differences in intensity levels introduced by different values of $\alpha$ and $\beta$. However, the results shown in
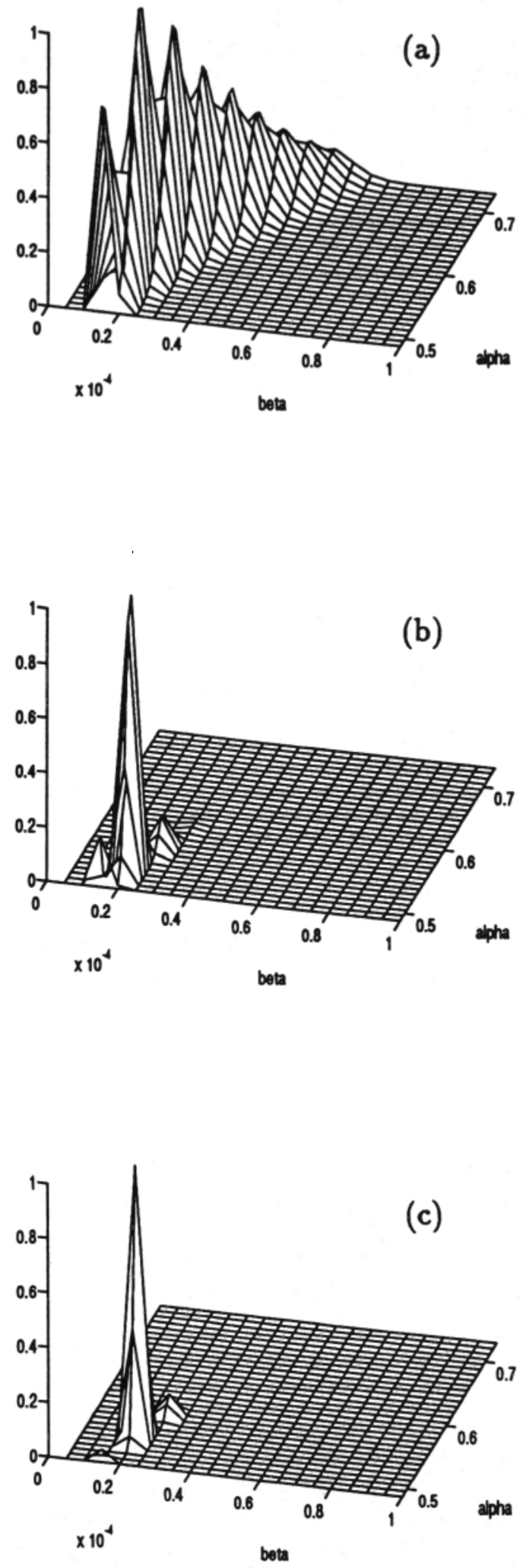

FIG. 2. Likelihood surfaces for simulated $\chi^{2}$ data with 2 dof for $\alpha=0.53$ and $\beta=2.0 \times 10^{-5}$ for (a) 10 , (b) 50 , and (c) 100 observations.

Tables I and II indicate that the point estimates obtained with and without normalization are very similar. This means that the results (i.e., point estimates) of the ML procedure are not significantly affected by the lack of calibration information. Therefore, this procedure can be ap- 
TABLE II. ML estimates for $\alpha$ and $\beta$ obtained from simulated backscatter with a $\chi^{2}$ modeling with 2 dof after normalization with respect to the first angle of incidence for 10, 50, and 100 observations.

\begin{tabular}{cccc}
\hline \hline & \multicolumn{3}{c}{ Maximum likelihood estimation of $\alpha$ and $\beta$} \\
\cline { 2 - 4 } Sample size & $\alpha=0.530$ & $\alpha=0.680$ & $\alpha=0.705$ \\
& $\beta=2.0 \times 10^{-5}$ & $\beta=4.0 \times 10^{-5}$ & $\beta=9.0 \times 10^{-5}$ \\
\hline 10 & 0.5883 & 0.7217 & 0.6383 \\
& $3.0 \times 10^{-5}$ & $5.0 \times 10^{-5}$ & $8.0 \times 10^{-5}$ \\
50 & 0.5217 & 0.6717 & 0.7050 \\
& $3.0 \times 10^{-5}$ & $4.0 \times 10^{-5}$ & $9.0 \times 10^{-5}$ \\
100 & 0.5300 & 0.6967 & 0.7050 \\
& $2.0 \times 10^{-5}$ & $4.5 \times 10^{-5}$ & $9.0 \times 10^{-5}$ \\
\hline \hline
\end{tabular}

plied to real data even when calibration information is not available.

Estimation after the application of the normalization scheme could prove problematic when the received field is scattered by a seafloor characterized by parameters $\alpha$ and $\beta$ significantly higher than the upper bounds of the grid used here. The angular dependence of backscatter decreases with increasing values of $\alpha$ and $\beta$, leading to relatively flat backscatter curves. Normalization of the received backscatter observations in cases of a small angular dependence would result in additional uncertainty in the estimation process. However, this problem does not arise for the ranges of interest of $\alpha$ and $\beta$, as shown by the results obtained for data generated for $\alpha=0.705$ and $\beta=9.0 \times 10^{-5}$, which are close to the upper bounds of the grid (Table II).

We also investigated the effect of averaging over a number of observations prior to the application of the ML estimation processor. It was thought that data smoothing could possibly lead to improved performance. This potential was tested using averages of 5 and 25 single observation vectors. Performing these averages leads to the creation of new rv's governed by scaled $\chi^{2}$ distributions with 10 and 50 dof, respectively. Tables III and IV contain the calculated estimates of $\alpha$ and $\beta$ for a $\chi^{2}$ modeling with 10 and 50 dof. The results indicate that averaging leads to an increase in accuracy. Tables $\mathrm{V}$ and VI show the corresponding estimates following normalization. Once again, it appears that the normalization procedure does not degrade the quality of the estimates. Figures 4 and 5 show the likelihood surfaces obtained from 50 observations with 2,10 , and 50 dof before and after normalization, respectively. It can be seen that increasing the number of dof leads to reduced uncertainty, manifested here as a decrease of the secondary modes. Thus, when possible, it is desirable to average over a number of observations before applying the ML processor.

\section{B. Real data}

Since the relationship between backscattered intensity and angle of incidence depends on the seafloor structure, multibeam bathymetric systems such as Sea Beam ${ }^{10}$ provide us with data in an ideal form for seafloor parameter
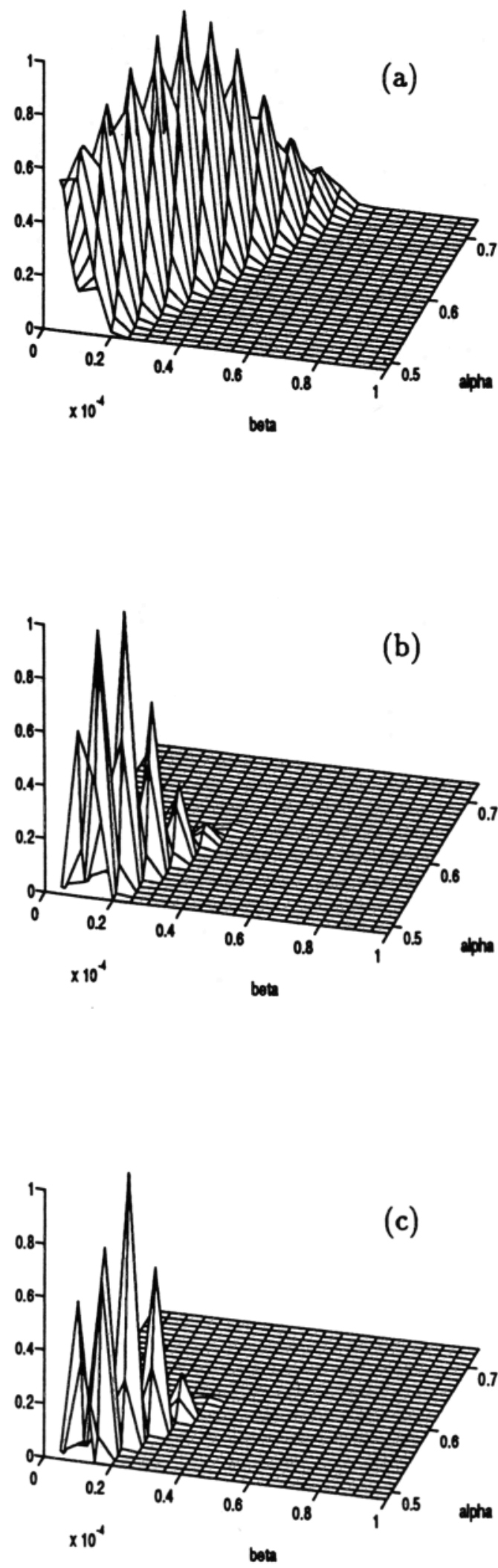

FIG. 3. Likelihood surfaces for simulated $\chi^{2}$ data with 2 dof for $\alpha=0.53$ and $\beta=2.0 \times 10^{-5}$ following normalization for (a) 10 , (b) 50 , and (c) 100 observations.

extraction. The Sea Beam system operates at $12.158 \mathrm{kHz}$ and receives acoustic backscatter through 16 preformed beams nominally spaced $\frac{80}{3}$ apart, between $\pm 20^{\circ}$ in athwartship angle relative to the ship's vertical axis. 
TABLE III. ML estimates for $\alpha$ and $\beta$ obtained from simulated backscatter with a $\chi^{2}$ modeling with 10 dof for 10,50 , and 100 observations.

\begin{tabular}{cccc}
\hline \hline & \multicolumn{3}{c}{ Maximum likelihood estimation of $\alpha$ and $\beta$} \\
\cline { 2 - 4 } Sample size & $\alpha=0.530$ & $\begin{array}{c}\alpha=0.680 \\
\alpha=2.0 \times 10^{-5}\end{array}$ & $\begin{array}{c}\alpha=4.0 \times 10^{-5} \\
\beta=9.0 \times 10^{-5}\end{array}$ \\
\hline 10 & 0.5717 & 0.7050 & 0.6800 \\
& $2.5 \times 10^{-5}$ & $4.0 \times 10^{-5}$ & $8.0 \times 10^{-5}$ \\
50 & 0.5300 & 0.6717 & 0.7050 \\
& $2.0 \times 10^{-5}$ & $4.5 \times 10^{-5}$ & $9.0 \times 10^{-5}$ \\
100 & 0.5300 & 0.6800 & 0.7050 \\
& $2.0 \times 10^{-5}$ & $4.0 \times 10^{-5}$ & $9.0 \times 10^{-5}$ \\
\hline \hline
\end{tabular}

Here, we use data recorded with Sea Beam over two central North Pacific seamounts, Horizon Guyot and Magellan Rise. In both cases the seafloor is heavily sedimented with similar morphology. ${ }^{11}$ The complex beamformed echoes collected by the Sea Beam system have been corrected for bottom slopes, beampattern geometry, and ship's roll to obtain backscattered intensity versus angle of incidence. ${ }^{1}$

The angular dependence of the measured intensity signals must be handled carefully. The ML processor is based on the premise that the angles of incidence can be assumed to be fixed and equal to the nominal beam directions for every ping. However, this assumption is violated by the movements of the ship carrying the system during the experiment. For this reason, before using the data sets as observations of pressure or intensity variables, we followed a data selection procedure, based on whether the actual angles of incidence for a ping, after the corrections reported by de Moustier and Alexandrou, ${ }^{1}$ fall in bins centered at the nominal angles $\theta_{i}$, where

$$
\theta_{i}=-20+\frac{8}{3}(i-1),
$$

where $i=1,2, \ldots, 16$. The bin size was selected here to equal $\frac{80}{3}$ centered around the $\theta_{i}$ 's of Eq. (16).

We work here with only the eight port beams. Following the binning procedure described above, we process only these pings for which the measured angles (after the corrections) fall inside the selected bins. The result of this procedure is the formulation of observation vectors of

TABLE IV. ML estimates for $\alpha$ and $\beta$ obtained from simulated backscatter with a $\chi^{2}$ modeling with 50 dof for 10,50 , and 100 observations.

\begin{tabular}{cccc}
\hline \hline & \multicolumn{3}{c}{ Maximum likelihood estimation of $\alpha$ and $\beta$} \\
\cline { 2 - 4 } Sample size & $\alpha=0.530$ & $\alpha=0.680$ & $\alpha=0.705$ \\
$\beta=2.0 \times 10^{-5}$ & $\beta=4.0 \times 10^{-5}$ & $\beta=9.0 \times 10^{-5}$ \\
\hline 10 & 0.5383 & 0.6800 & 0.6967 \\
& $2.0 \times 10^{-5}$ & $4.0 \times 10^{-5}$ & $9.0 \times 10^{-5}$ \\
50 & 0.5300 & 0.6800 & 0.7050 \\
& $2.0 \times 10^{-5}$ & $4.0 \times 10^{-5}$ & $9.0 \times 10^{-5}$ \\
100 & 0.5300 & 0.6800 & 0.7050 \\
& $2.0 \times 10^{-5}$ & $4.0 \times 10^{-5}$ & $9.0 \times 10^{-5}$ \\
\hline \hline
\end{tabular}

TABLE V. ML estimates for $\alpha$ and $\beta$ obtained from simulated backscatter with a $\chi^{2}$ modeling with 10 dof after normalization with respect to the first angle of incidence for 10,50 , and 100 observations.

\begin{tabular}{cccc}
\hline \hline & \multicolumn{3}{c}{ Maximum likelihood estimation of $\alpha$ and $\beta$} \\
\cline { 2 - 4 } Sample size & $\alpha=0.530$ & $\alpha=0.680$ & $\alpha=0.705$ \\
$\beta=2.0 \times 10^{-5}$ & $\beta=4.0 \times 10^{-5}$ & $\beta=9.0 \times 10^{-5}$ \\
\hline 10 & 0.555 & 0.6717 & 0.6967 \\
& $2.5 \times 10^{-5}$ & $4.0 \times 10^{-5}$ & $9.0 \times 10^{-5}$ \\
50 & 0.5300 & 0.6800 & 0.7050 \\
& $2.5 \times 10^{-5}$ & $4.0 \times 10^{-5}$ & $9.0 \times 10^{-5}$ \\
100 & 0.5300 & 0.6800 & 0.7050 \\
& $2.0 \times 10^{-5}$ & $4.0 \times 10^{-5}$ & $9.0 \times 10^{-5}$ \\
\hline \hline
\end{tabular}

length 8 , the elements of which have been measured close to the nominal steering angles within a margin of tolerance defined by the bin size.

The objective here is to produce ML estimates of the parameters $\alpha$ and $\beta$ based on the available Sea Beam data. These same data were previously analyzed from an estimation point of view by using a curve-fitting method, attempting to match the angular dependence formed by the observations at the two regions to the model of Sec. I. ${ }^{1}$ An exact fit was not found for either set. There were indications, however, that the observed curves were satisfactorily close (especially for Horizon Guyot) to the theoretical ones for two different pairs of $\alpha$ and $\beta$ estimates, where each pair of estimates corresponds to different ranges of angles of incidence. The ML estimator employed here attempts to find a global estimate (i.e., covering the whole range of angles of incidence) for each seafloor region. Therefore, some differences from the curve fitting results ${ }^{1}$ are to be expected.

The ML processor requires information concerning the distributions of the variables of interest. The distribution of instantaneous intensity for a specific angle of incidence is of a scaled $\chi^{2}$ form, as has been previously explained. Independence among observations from both adjacent angles and pings is assumed. Correlation coefficients have been actually calculated and found to vary between -0.2 and 0.26 for observations from both adjacent beams and consecutive pings. The modeling of the likelihood function follows from Eqs. (12) and (13), and the normalization scheme discussed in Sec. IV A is used be-

TABLE VI. ML estimates for $\alpha$ and $\beta$ obtained from simulated backscatter with a $\chi^{2}$ modeling with 50 dof after normalization with respect to the first angle of incidence for 10,50 , and 100 observations.

\begin{tabular}{cccc}
\hline \hline & \multicolumn{3}{c}{ Maximum likelihood estimation of $\alpha$ and $\beta$} \\
\cline { 2 - 4 } Sample size & $\alpha=0.530$ & $\alpha=0.680$ & $\alpha=0.705$ \\
& $\beta=2.0 \times 10^{-5}$ & $\beta=4.0 \times 10^{-5}$ & $\beta=9.0 \times 10^{-5}$ \\
\hline 10 & 0.5550 & 0.6800 & 0.7050 \\
& $2.5 \times 10^{-5}$ & $4.0 \times 10^{-5}$ & $9.0 \times 10^{-5}$ \\
50 & 0.5300 & 0.6800 & 0.7050 \\
& $2.0 \times 10^{-5}$ & $4.0 \times 10^{-5}$ & $9.0 \times 10^{-5}$ \\
100 & 0.5300 & 0.6800 & 0.7050 \\
& $2.0 \times 10^{-5}$ & $4.0 \times 10^{-5}$ & $9.0 \times 10^{-5}$ \\
\hline \hline
\end{tabular}



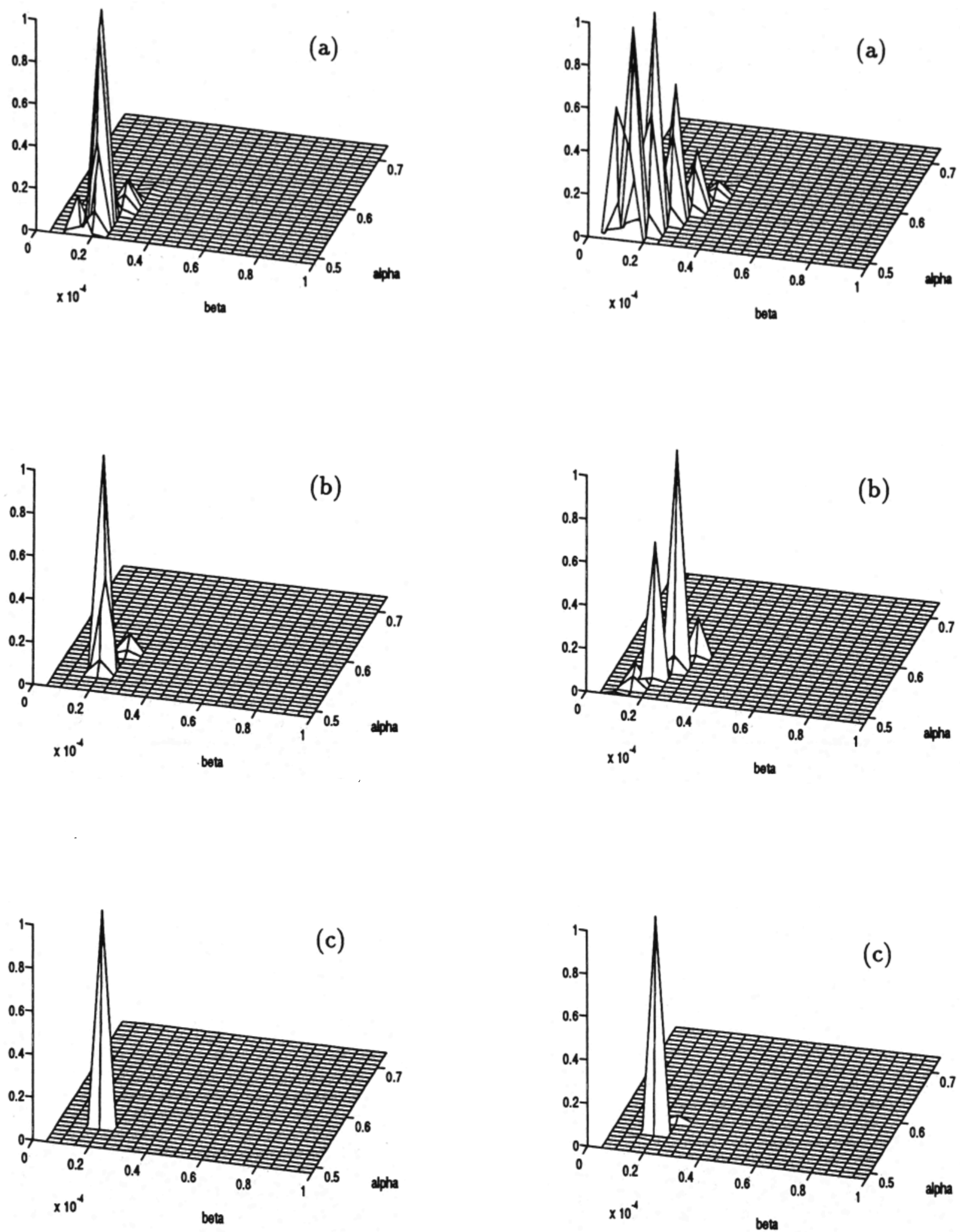

FIG. 4. Likelihood surfaces for 50 observations of simulated $\chi^{2}$ data with (a) 2 , (b) 10 , and (c) 50 dof for $\alpha=0.53$ and $\beta=2.0 \times 10^{-5}$.

cause the Sea Beam measurements were not calibrated. Following the binning procedure, there are 425 available observations for Horizon Guyot and 124 for Magellan Rise.

Ideally, it is desirable to process average instead of

FIG. 5. Likelihood surfaces for 50 observations of simulated $\chi^{2}$ data with (a) 2 , (b) 10 , and (c) 50 dof for $\alpha=0.53$ and $\beta=2.0 \times 10^{-5}$ following normalization.

instantaneous intensities within each beam for a specific ping. The number of samples within a beam varies with the angle of incidence, i.e., fewer measurements are obtained in the near-vertical beams, whereas more observations are 
TABLE VII. ML estimates for the Horizon Guyot region obtained for $10,50,100$, and 425 observations.

\begin{tabular}{ccc}
\hline \hline \multirow{2}{*}{ Sample size } & \multicolumn{2}{c}{ Maximum likelihood estimation of $\alpha$ and $\beta$} \\
\cline { 2 - 3 } 10 & $\chi^{2}$ rv's (2 dof) & $\chi^{2}$ rv's (10 dof) \\
\hline \multirow{2}{*}{50} & 0.5883 & 0.6467 \\
& $3.0 \times 10^{-5}$ & $4.5 \times 10^{-5}$ \\
100 & 0.6467 & 0.6383 \\
& $4.5 \times 10^{-5}$ & $4.5 \times 10^{-5}$ \\
425 & 0.6217 & - \\
& $4.0 \times 10^{-5}$ & - \\
& 0.6133 & - \\
\hline \hline
\end{tabular}

available in the outer beams. ${ }^{1}$ Thus averaging over different numbers of observations would require the construction of distributions with a varying number of dof from beam to beam, leading to a more complicated problem. In addition, averaging within each beam would require a test of independence between consecutive samples. For these reasons, we currently use a single measurement per beam, obtained at the expected center of the beam.

On the other hand, returns from different pings have been found to be independent. Thus averaging over instantaneous intensity observations obtained from several consecutive pings is performed. The $\chi^{2}$ modeling with more than 2 dof as described in Sec. II is necessary in this case. Here, we average observations from five consecutive pings leading to $\chi^{2}$ rv's with 10 dof. It may be argued that averaging over a large number of pings would be desirable as it would lead to additional smoothing of the data. However, averaging over a large number of pings is not recommended, unless a certain degree of spatial homogeneity of the region under investigation is guaranteed.

Table VII presents the estimates for parameters $\alpha$ and $\beta$ for Horizon Guyot obtained through $\chi^{2}$ modeling with 2 and 10 dof. Representative likelihood surfaces are shown in Fig. 6. Examining the results of Table VII, one could conclude that for this data set $\alpha$ is between 0.61 and 0.64 and $\beta$ is close to $4.5 \times 10^{-5}$. It should be noted that the quality of the estimates is not affected when the ML processor is applied to a relatively small number of samples (e.g., 50). This is a useful feature when surveying complex terrains.

Curve fitting applied to the same data set produced estimates of 0.59 and $4.0 \times 10^{-5} .{ }^{1}$ It must be recalled here that two different estimates were reported by de Moustier and Alexandrou ${ }^{1}$ for the same data. The two pairs of estimates corresponded to the angular ranges $0-6^{\circ}$ and $5-20^{\circ}$, respectively. Because of variance constraints on the data near nadir, the solution corresponding to larger angles of incidence had been selected ( $\alpha=0.59$ and $\left.\beta=4.0 \times 10^{-5}\right)$. Again, we expect our results to differ somewhat because the ML processor relies on the entire angular range, whereas curve fitting emphasizes the outer angles. It should also be noted that the likelihood surface in Fig. 6(a) exhibits a very strong secondary mode, peaking
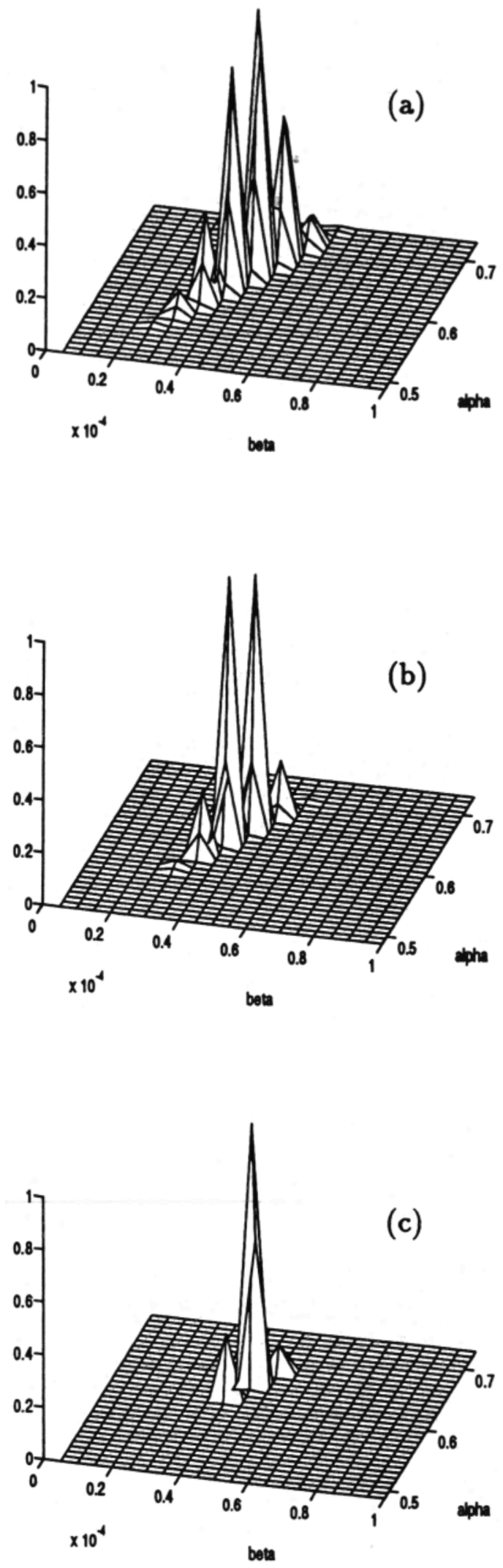

FIG. 6. Likelihood surfaces for Horizon Guyot with a $\chi^{2}$ modeling with 2 dof for (a) 50 and (b) 100 observations and (c) with 10 dof for 50 observations. 
TABLE VIII. ML estimates for the Magellan Rise region obtained for 10, 50, and 100 observations.

\begin{tabular}{ccc}
\hline \hline \multirow{2}{*}{ Sample size } & \multicolumn{2}{c}{ Maximum likelihood estimation of $\alpha$ and $\beta$} \\
\cline { 2 - 3 } & $\chi^{2}$ rv's (2 dof) & $\chi^{2}$ rv's (10 dof) \\
\hline 10 & 0.5217 & 0.4883 \\
& $5.0 \times 10^{-6}$ & $1.5 \times 10^{-5}$ \\
50 & 0.4967 & - \\
& $5.0 \times 10^{-6}$ & - \\
100 & 0.5050 & - \\
& $5.0 \times 10^{-6}$ & - \\
\hline
\end{tabular}

around $\alpha=0.60$ and $\beta=4.0 \times 10^{-5}$, which are the dominating curve fitting estimates. ${ }^{1}$

Table VIII and Fig. 7 present the results obtained for the Magellan Rise data by following the same procedure. Accepting that modeling with more dof produces more reliable results, we select the estimates for $\alpha$ and $\beta$ close to 0.49 and $1.5 \times 10^{-5}$, respectively. All figures show the likelihood surfaces to be mostly concentrated at a corner of the grid. Although it is possible that the correct estimates in fact happen to be located at the corner, the results suggest that the grid, selected based on our prior information concerning $\alpha$ and $\beta$, may not be broad enough to cover the range of the parameters valid for this seafloor region. Theoretically, it would be possible to extend the grid around the area of the peak and carry out a new search for a maximum. In this specific problem, however, this would violate the assumptions of the acoustic model, according to which the range of $\alpha$ does not extend below $0.5{ }^{2}$

For the Magellan Rise data, the ML estimation results differ from the curve fitting estimates, ${ }^{1}$ where $\alpha$ and $\beta$ were found to be equal to 0.55 and $3.5 \times 10^{-5}$. However, it should be noted that the curve-fitting procedure applied to these data did not yield a very close match to the theoretical backscattering strength curves. It could be that the chosen acoustic model is not appropriate for these data, particularly with regard to potential volume scattering contributions. Although core samples taken in each area revealed similar surficial lithologies of foraminiferal ooze with high calcium carbonate content, the sediments were more consolidated on Horizon Guyot than on Magellan Rise. In addition, the Magellan Rise site is twice as deep as the Horizon Guyot site. Although every effort was made to render the measurements depth independent by accounting for transmission losses in the water column and the area insonified on the bottom for each beam, the measurements made over Magellan Rise have comparatively smaller signal-to-noise ratios, in part because of higher sea states at the time of data acquisition. These smaller signal-to-noise ratios coupled with a smaller set of samples available for the Magellan Rise area may have contributed to the poor fit and the discrepancies observed.

\section{CONCLUSIONS}

The ML estimation method suggested in this paper appears to be a readily applicable tool in the context of
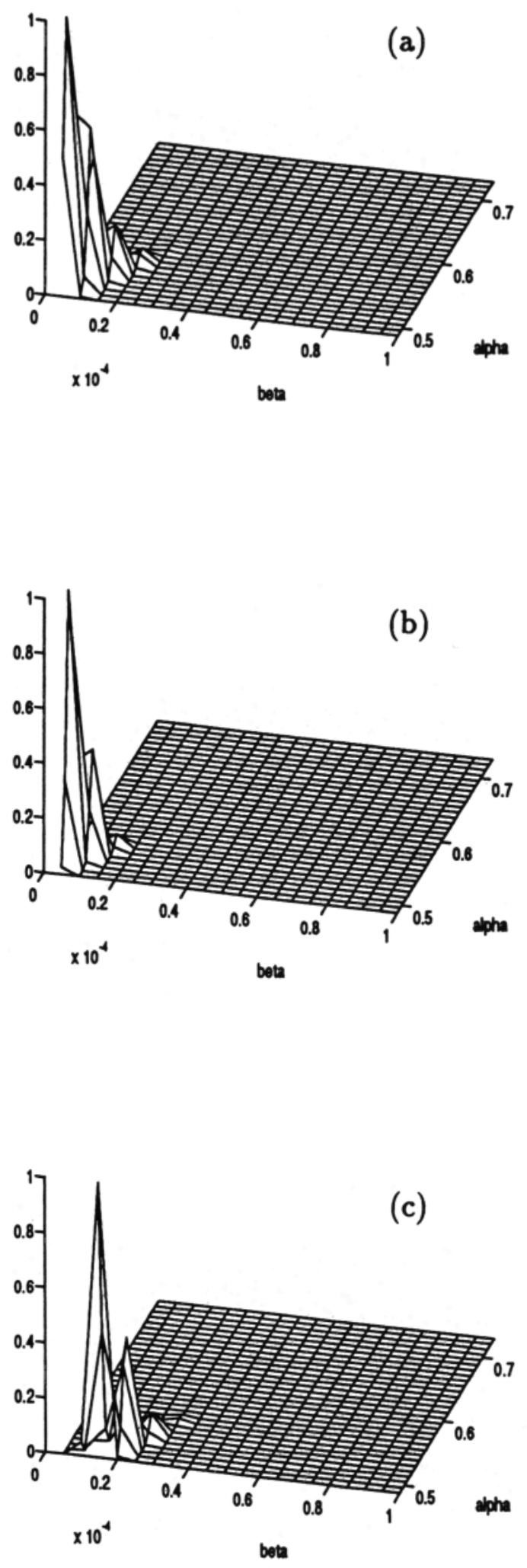

FIG. 7. Likelihood surfaces for Magellan Rise with a $\chi^{2}$ modeling with 2 dof for (a) 50 and (b) 100 observations and (c) with 10 dof for 10 observations.

seafloor parameter estimation based on real data, provided the data fit the selected acoustic model. Our technique has a number of advantages over the curve fitting method. ${ }^{1}$ The ML processor is based on a quantitative performance 
criterion as opposed to a qualitative goodness-of-fit and thus it can be highly automated. In addition, the ML method takes advantage of known statistical characteristics of bottom reverberation. As a result, it is more efficient, requiring a significantly smaller number of acoustic records. Finally, the likelihood surfaces provide useful insight into the uncertainty associated with the estimation process.

The difficulty of estimating seafloor parameters from the Magellan data set points to potential modeling problems. The ML estimation method can be refined by incorporating into the model the influence of ambient noise and the presence of a coherent component in the near-nadir observations. The potential contribution of volume scattering can also be included. In addition, efforts are currently underway to design a processor able to handle angular uncertainty. Such a processor would eliminate the need for the data selection process discussed in Sec. IV B and would make more efficient use of Sea Beam measurements.

\section{ACKNOWLEDGMENTS}

This work was funded by the Office of Naval Research, Code 1125GG, through Contract No. N00014-93-I-0049.
${ }^{1}$ C. de Moustier and D. Alexandrou, "Angular dependence of 12-kHz seafloor acoustic backscatter," J. Acoust. Soc. Am. 90, 522-531 (1991).

${ }^{2}$ D. R. Jackson, D. P. Winebrenner, and A. Ishimaru, "Application of the composite roughness model to high-frequency bottom backscattering," J. Acoust. Soc. Am. 79, 1410-1422 (1986).

${ }^{3}$ H. Matsumoto, R. P. Dziak, and C. Fox, "Estimation of seafloor microtopographic roughness through modeling of acoustic backscatter data recorded by multi-beam systems," J. Acoust. Soc. Am. 94, 27762787 (1993).

${ }^{4}$ G. E. P. Box and G. C. Tiao, Bayesian Inference in Statistical Analysis (Addison-Wesley, Reading, MA, 1973), Chap. 5, pp. 244-316.

${ }^{5}$ R. J. Urick, Principles of Undenwater Sound (McGraw-Hill, New York, 1983), Chap. 8, pp. 211-262.

${ }^{6}$ C. G. Fox and D. E. Hayes, "Quantitative methods for analyzing the roughness of the seafloor," Rev. Geophys. 23, 1-48 (1985).

${ }^{7}$ V. V. Ol'shevskii, Statistical Methods in Sonar (Consultants Bureau, New York, 1978), Chap. 6, pp. 107-146.

${ }^{8}$ V. V. Ol'shevskii, Characteristics of Sea Reverberation (Plenum, New York, 1967), Chap. 3, pp. 51-72.

${ }^{9}$ W. S. Burdic, Underwater Acoustic System Analysis (Prentice-Hall, Englewood Cliffs, NJ, 1984), Chap. 12, pp. 361-379.

${ }^{10}$ V. Renard and J. P. Allenou, "SEA BEAM multi-beam echo-sounding in 'Jean Charcot.' Description, evaluation and first results," Int. Hydro. Rev. LVI, 35-67 (1979).

${ }^{11}$ K. L. Smith, W. C. Schwab, M. Noble, and C. de Moustier, "Physical, geological, and biological studies on four Pacific seamounts: Introduction," Deep-Sea Res. 36, 1785-1790 (1990). 